\title{
RACCOLTA DI DATI IONOSFERICI DEDOTTI DA PROVE DIRETTE DI COLLEGAMENTI R.T. EFFETTUATI SULLA RETE DELL'ESERCITO
}

\author{
Stelio Silleyi
}

\section{PART: II}

1. Generalitio, - Dall'anosto 1948 al seltembre 1949 sono stati raccolti i dati di esercizio di una rete R.T. che utilizza generalmente la propagazione ionoferica: questi dati sono sati confrontati con quelli dei sontlagyi ionosferici sistematici effettuati presso l'Istituto Nazionale di Geofisiea, in Roma.

Nella parte I di questo suclio 1 1, si i riferito sul metodo adottato per l'elaborazione e sll risultati ottenuli in alcuni mesi di sperimentazione. Si danno ora yli ulteriori riwultati e le conclusioni finali. Per la descrizione particolareguiata del metodo e per molti chiarimenti e con-iderazioni si rinvia alla Parte I suddetta.

Aleune notazioni sono state modificate per uniformarsi con quelle ormat adottate in sede internazionale (C.C.I.R.)

-- WIV = frequenza mas-ima usabile, già indicata con MFU; FOT = frequenza ottima di lavoro convenzionalel gìi indicala con FOL.

2. Dati eloro elaborazione. - L'elaborazione dei dati ì stata fatta secondo il metodo macrotatistien descritlo nella parte I (n. 5).

$\mathrm{Si} i$ anche provato ad applieare un metodo hasato sui minimi quadrati. Per ciaceun mese sono sali riporlati i punti (f MUF: probabilitil : $=$ un piano ad una coordinata trasformata per anamorfosi come quello di fig. L. Lale ehe qualora la distribuzione fose gaussiana. si arrelle una retta. Su tale piano sono state dedotte le coordinate di una relta che segue l'andamento dei punti rendendo minimo l'errore quadratieo medio. Per confrontare i due metodi si a calenlato per ciascuno di eni il coefficiente di correlazione dei risultati ot-

11, Annali di Geoficicil. vol. II. n. 3, parer. $388-399$ (luglio 1919).

i- Comite Consultatil International des Radiocommunications. 


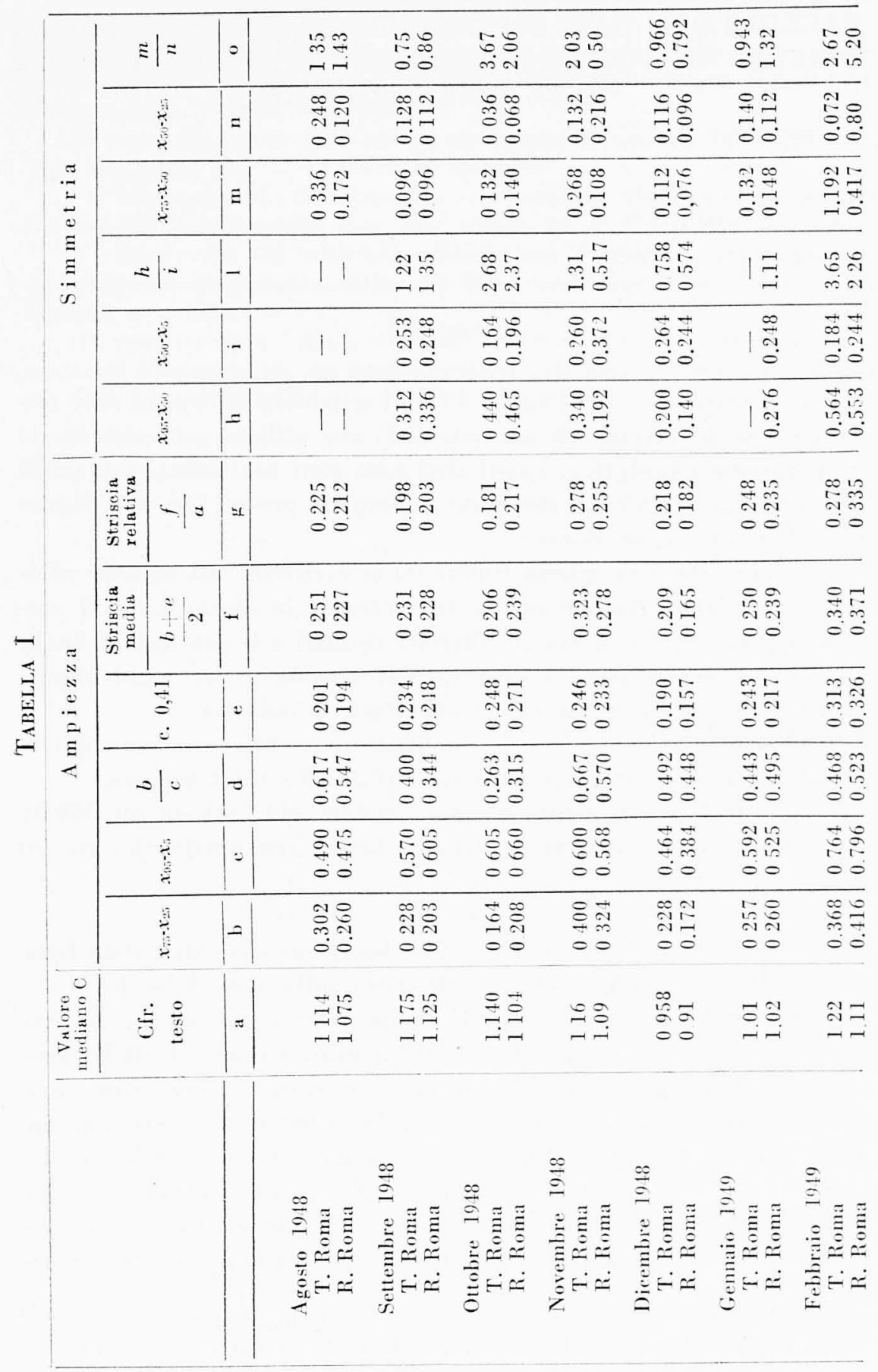


RACCULTA DI DATI IONOSFERICI DEDOTTI DA PROVE DIRETTE DI COLLEGAMENTI R. T. 569

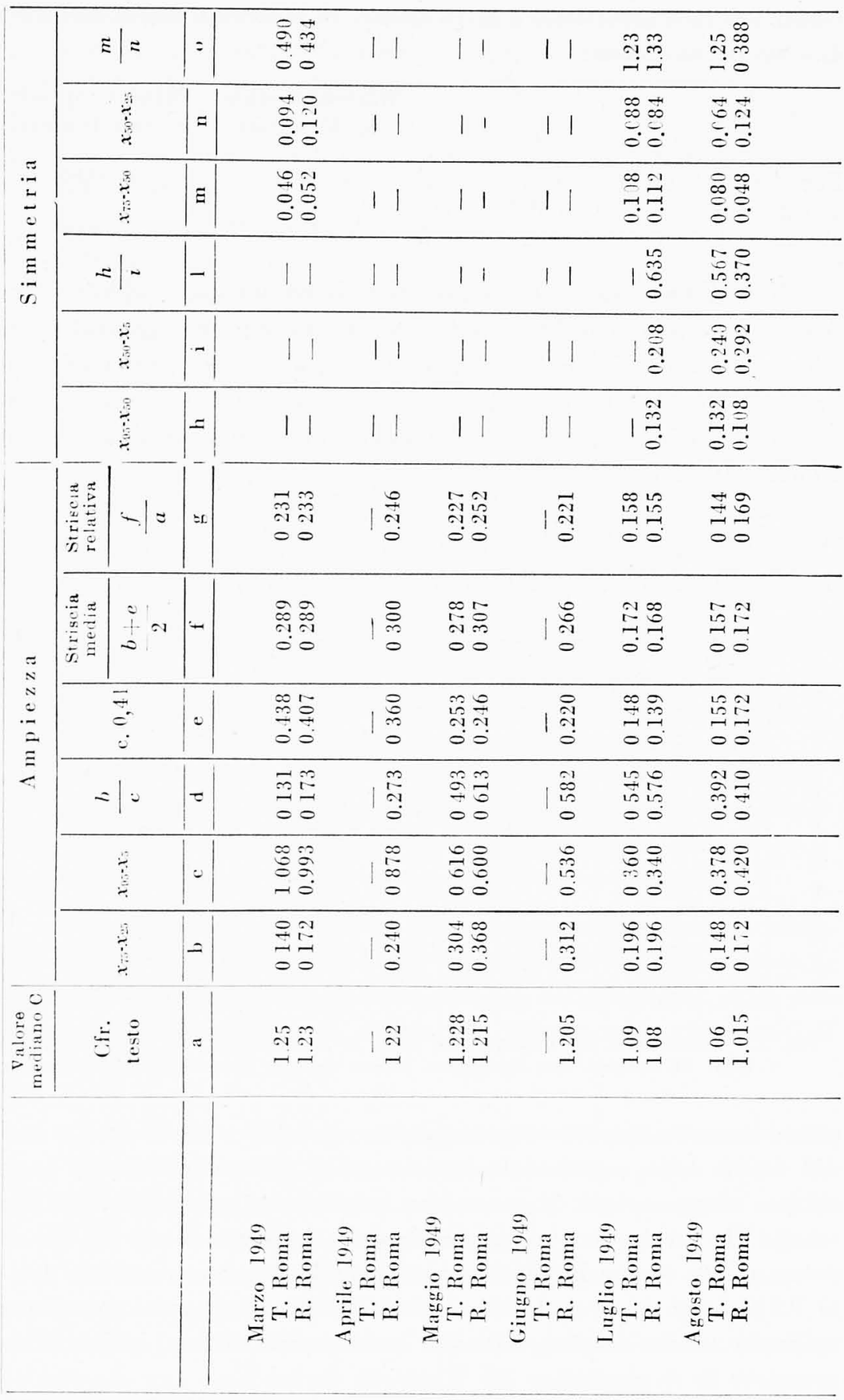


lenuli per la trasmiswione e la ricezione, in ciaseun mese. I rocflicienli di correlazione sono:

$$
\begin{gathered}
\text { Macrostatistico Minimi quatrati } \\
\text { (su } 13 \text { mesi) }
\end{gathered}
$$

Per il valore mediano C. . . . .

Per l'ampiezza relativa della stri-

$$
\text { seial del 50\%\%. . . . . . . . . } 50.80 \quad+0.85
$$

Yon si nota una nelta superiorita di un metodo sull'altro. Se si tiene conto peró che il metorlo basallo sui minimi quadrati i più laborioso e di mageviore reso ai dati estremi. che in realta non somo i pliù signifeativi. la bilancia si snosta a favore del metodo macrostalistico, la cui efficienza i più che suffeciente se si considera il grande numero di dati elaborati.

3. Effetlo della rariazione di potenza. - Tei due versi thatsmissione da Roma-ricezione da Romal e per le frequenze più hata-s somen

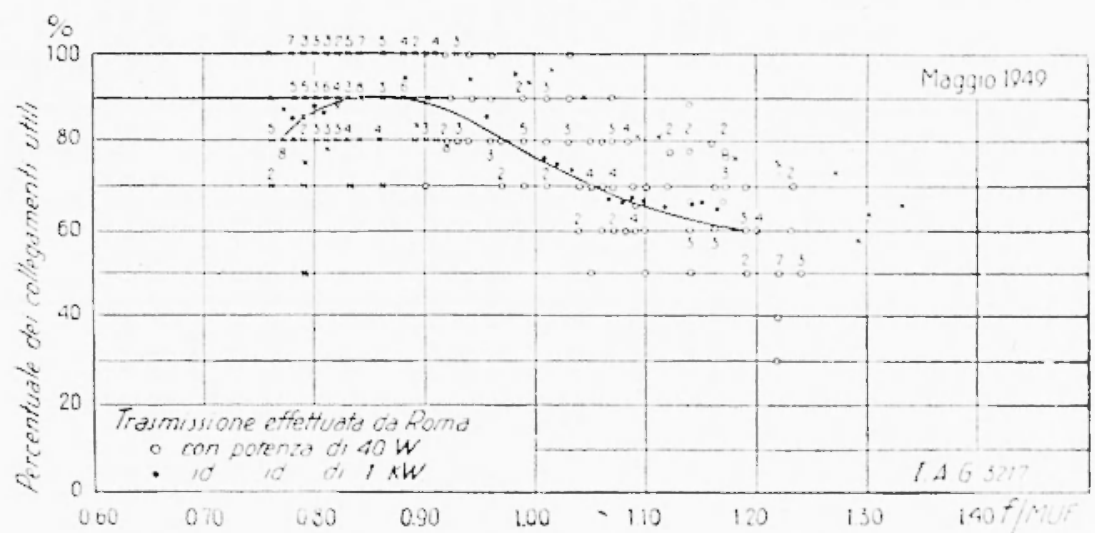

Figr. I - Percentuale dei collegamenti utili in trismissione dil Roma - maggio 19.19. Si osserva una frequenza ollima nguale a cirea $0.8 \%$ tht li.

state impiegate rispettivamente potenze di $1 \mathrm{k} / \mathrm{l}$ c di $40 \mathrm{~W}$. Lo searto dal 100\% della percentuale massima dei collenamenti utili i stato sempre maggiore per il verso che impiega la potenza minore fofre tabella Jl). In media, la variazione della potenza da $10 \mathrm{~W}$ a $1 . \mathrm{k} f$ porta questo scarto, in corrispondenza della frequenza ottina, dal 2,6 al 9,9\%; cioe la probabilitì che un collegamento non posia venire utilizzato, anche se progettato per la frequenzal ottina, i circal t volte maggiore se si impiegano 40 II invece di $1 \mathrm{kll}$. 
Per lrequenze più basse di quella oltima l'influenza della potenza dovelbhe essere maggiore. Per frequenze più alte essa i Irascurabile, entro cerli limili, rispelto alla influenza della variazione di frefuenza. I diagrammi delle lig. 1 e 2 traceiati per il mese di magrgio $19+9$, damno una indicazione ablastanza evidente di questo andanento. Su di essi infilti si nola:

- lo scarlo dal $100 \%$ è più basso quando la polenza è più alı;

- la frefuenza ollima e più lassa quando la polenza è più alta;

- per frequenze decrescenti a parlire da quella oltima la diflerenza delle probabiliti tende ad aumentare;

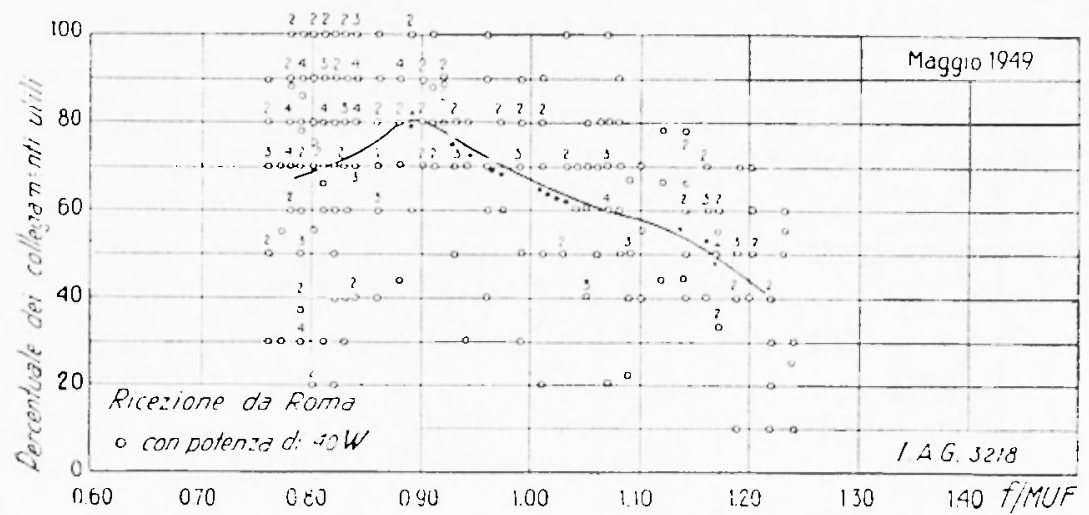

Fiy. 2- Perrentuale dei collegamenti utili in ricezione da Roma - maggio 1949. Si osiersa una frequenza oltima uguale a circa 0,89 MLF.

- per frequenze cresenti a partire da quella ottima la differenza delle prohalibliti si mantiene approssimatianente rostante, e quindi acquista importanza sempre minore relativamente alla probabilita di collewamento non ulilizzal,ile dovuto alla vitriazione di frepuenza.

1. Effelto della variazione di jrequenza. - I valori medi della slriscia del $50 \%$ calcolati su tutto l'amo risultano poco diversi da quelli calcolati nella parte J. La media amma risulta uguale a 0,22 dellat WIF, con flutuazioni abbatsanza forti.

La correlazione fra l'ampieza degli searti dedotti dai collegamenti

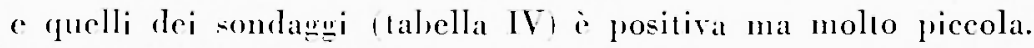

Cio indica che lat flutuazione delle frequenze eritiche (sondaggil i soltanto una componente di quella dei collegamenti. Il diagram- 
Tablela II

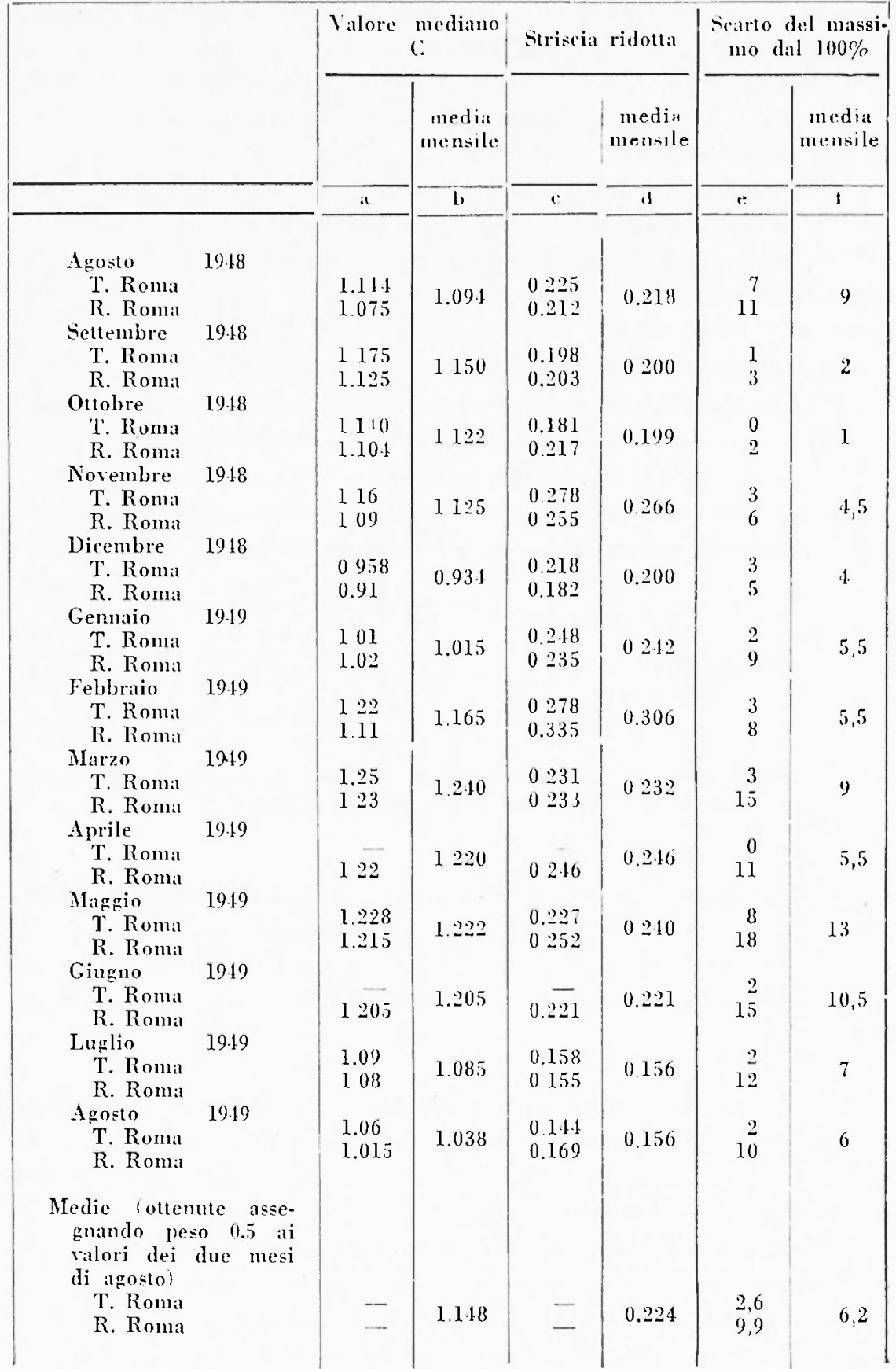




\section{Timela III}

\begin{tabular}{|c|c|c|c|c|}
\hline \multicolumn{2}{|c|}{ Mese } & $\begin{array}{l}\text { Valore mediano } \\
\text { oftenuto con la }\end{array}$ & $\begin{array}{l}\text { Valore mediano } \\
\text { dedotto dei col- }\end{array}$ & Scarto \\
\hline Anosio & 19.18 & & 1094 & \multirow{14}{*}{ 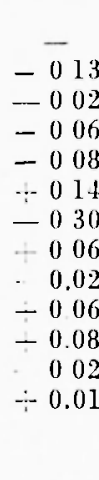 } \\
\hline Setteml,re & 19.48 & 1.28 & 1.150 & \\
\hline Ottobre & 19.48 & 111 & 1122 & \\
\hline Novembre & 19.48 & 1.18 & 1125 & \\
\hline Dicembre & 19.48 & 1.01 & 0.934 & \\
\hline Gemmaio & 1919 & 0.88 & 1015 & \\
\hline Febluaio & 19.19 & 1.7 & 1165 & \\
\hline Marzo & 19.19 & 1.10 & 1240 & \\
\hline Aprile & 19.49 & 124 & 1220 & \\
\hline Magrio & 19. 19 & 1.11 & 1222 & \\
\hline (iinguo & 19.49 & 1.13 & 1205 & \\
\hline Isuplio & L9:19 & 111 & 108.5 & \\
\hline Agoto & $19.4^{\prime}$ & 103 & 1.038 & \\
\hline \multicolumn{2}{|l|}{ Media } & 11.48 & 1.127 & \\
\hline eluzione & & & & \\
\hline
\end{tabular}

\section{Tabelea IV}

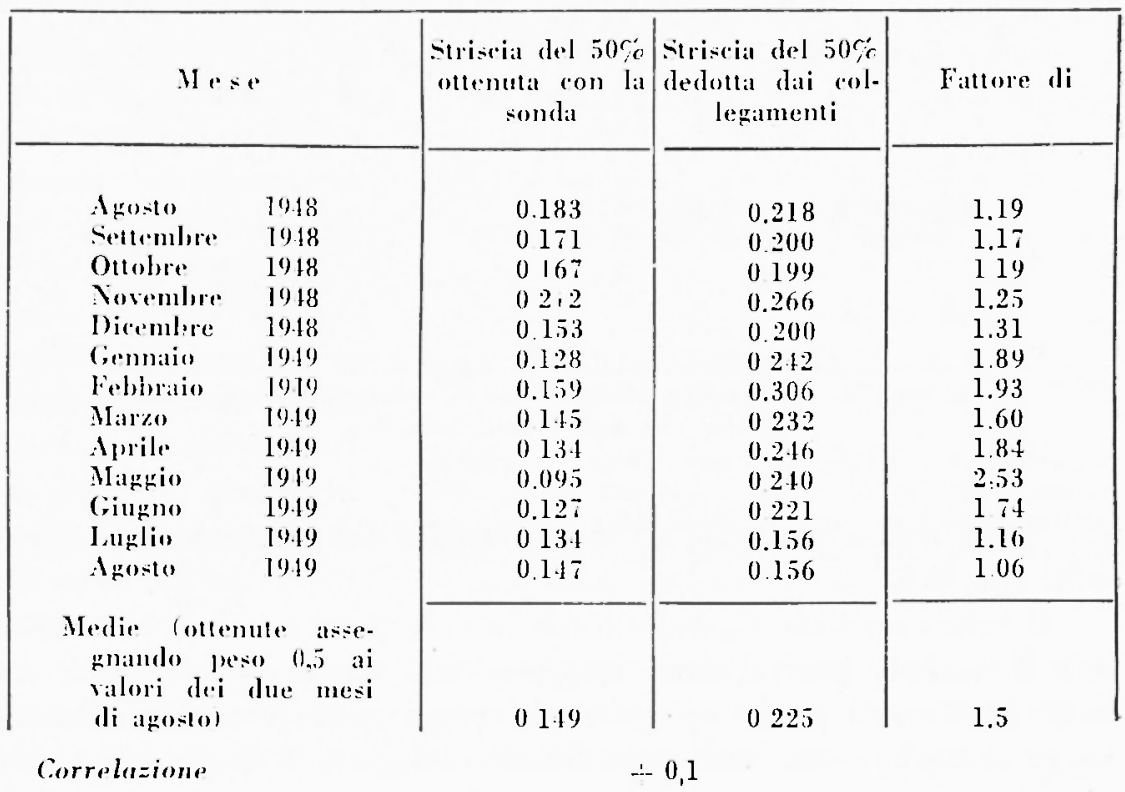


ma della fig̣. 3 mostra una interessante particolarita di questa relatxione: per la prima meta dell'amno il rapporto tra le due flutuazioni è molto magyiore che per la seconta meta lell'amno. Le imiagini finora falle non hanno indieato nessuna causa fisica di un tale comportamento. Non i escluso clue tale causa possa trovarsi in altri campi,

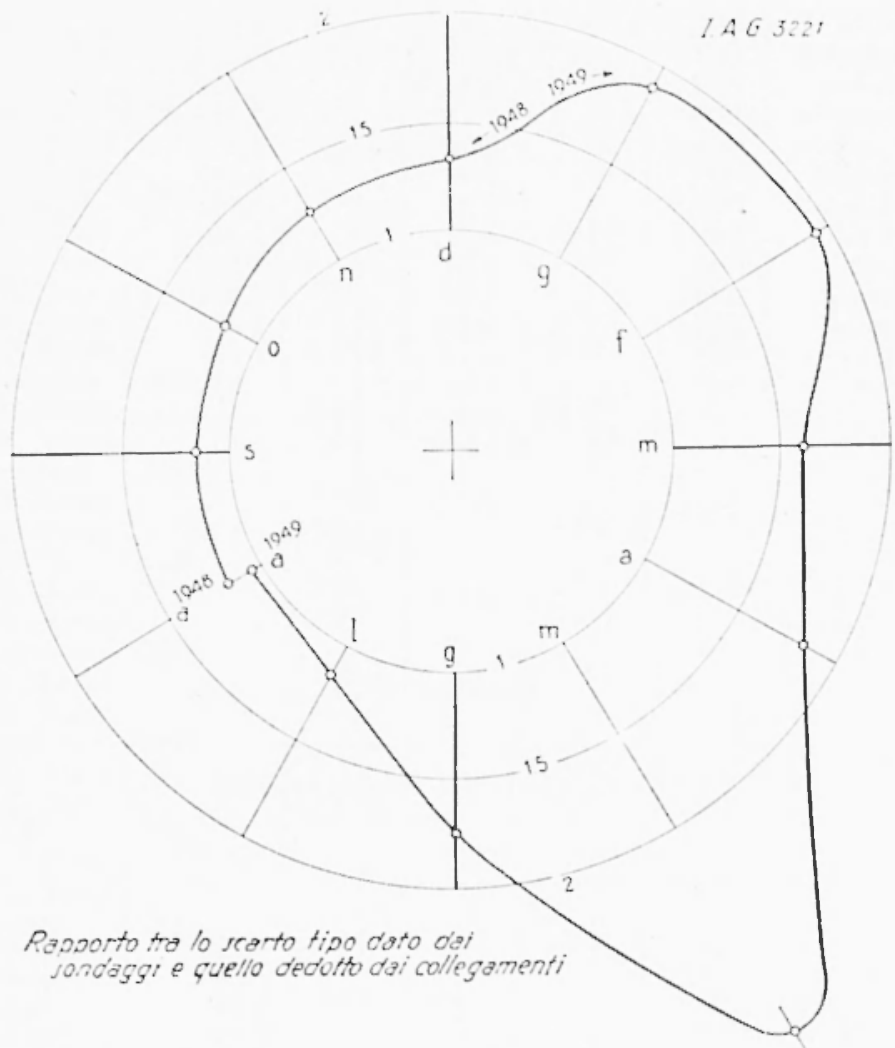

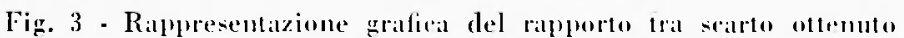

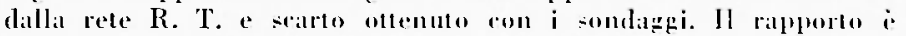
quello che figurat nellit tab. W.

p. e. prossal venire attrilunita all'anlilestramento del personale impiegato sullal rete R.T.

Il valore merliano $C$, leslote con le avertenze indicate nella parte I, è risultato eneneralmente maggione di l eal in menlia $i$ stato di ],] 18. Durante lo stesso intervallo di tempo anche $i$ valori misurati con la ionosonda sono slati generalmente maggiori di quelli delle pue. 
visioni CRPL-D. La correlazione dei valori mensili (tahella Mll) di questi coeffieienti i 0,6 , cioe molto alta se si osserva che la correlazione degli stessi coeffieienti falla solo per la rete R.T., tra dati di ricezione e di trasmissione, $\grave{e}$ di $0,7 \mathrm{l}$. E interessante notare che durante questo tempo l'attiviti solare is stata anch'essa generalmente più clevata di quella prevista nelle publolicazioni citate; in merlia il rapporto tra media molile dei numeri di Wolf osservati da Zurigo fatta sul 12 mexi e numero di IVoll previsto eal adottato dalle publilicazioni CRPL-D is stato di I.21.

La distriluzione relle pereentuali dei collewamenti utili si presentata generalmento asimmotrical tabella 1). Crescendo la Lre. quenza al di sopra alella MIL fe le pereentuali ratlono piò rapislamente di fuanto exse non arescano diminuendo la frequenza al di sotlo dellat MUT. Questo andimonto i analogero a quello rhe si osecrva nei somlagwai, nei fuali in presenza di forti perturlazioni ionosfericlue si lamo valori della frecpuenza critica molto più basid di quel. li mediani. mentre invere is acergionale osservare valori molto

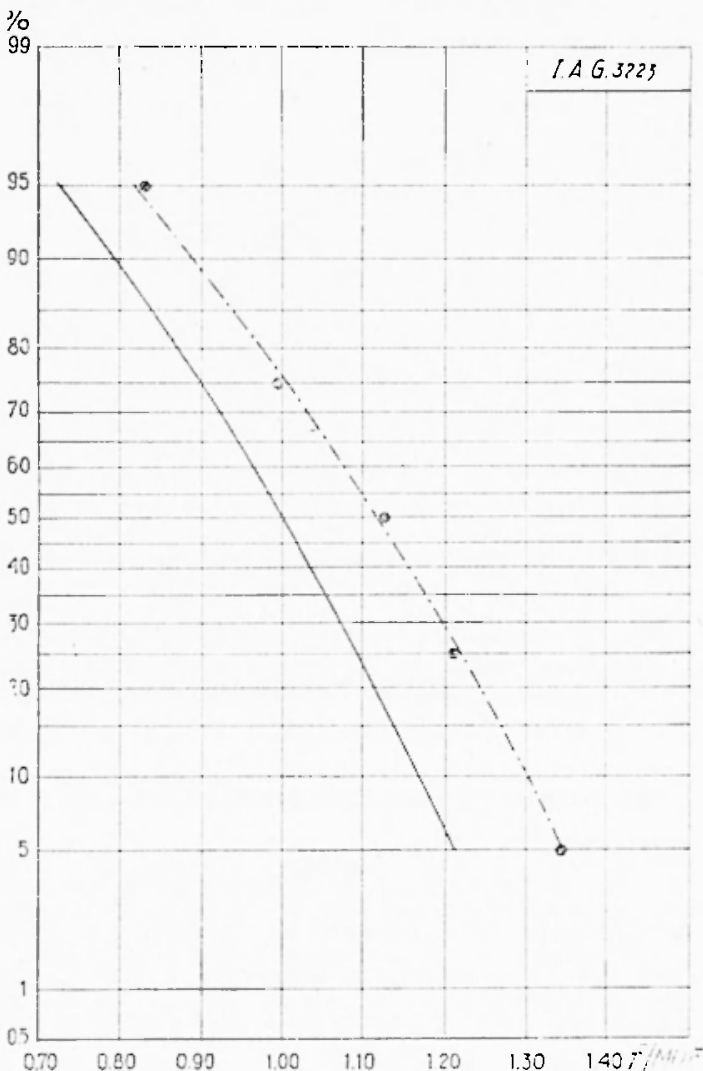

Fig. t - I valori medii di I/MUF corrispondenti alle probabiliti del $5,25,50,5,5$ e 95, c. riportati su carta di probabiliti. indicano una distriburione non gillssiana. La curva a tratto pieno è affine alla precedente, mal pissal per il punto $11 ; 00 \mathrm{c} / \mathrm{cl}$ piì alıi.

Per oservare menlio fuesto particolare, e tenerne conto, si i trace $\therefore$ ata lat curval a tratto e punto della fig. 1, in cui l'asse verticale $e$ eradualo secondo l'integrale della fumzione degli errori. I punti ripor- 
tati sono quelli che hamno come ascissa le medie dei valori mensili f MUF corrispondenti alla stessa percentuale. Se la distribuzione fosse gaussiana si dorrebbe avere una retla, mentre invere si ottiene ma curva con la concavià in hasso. La curva a tralto pieno i ollenula da quella a trallo e punto per affinita, rispelto all'asse verlicale, con un coefficiente tale da portare la probabilita del $50 \%$, in corrispondenzal di $\mathrm{f} / \mathrm{MUF}=1$.

La stessa legge ¿̀ rappresentata nel diagramma 5 in cui le coordinate sono lineari. La curva tratlegrgiala di questo diagramma rappre-

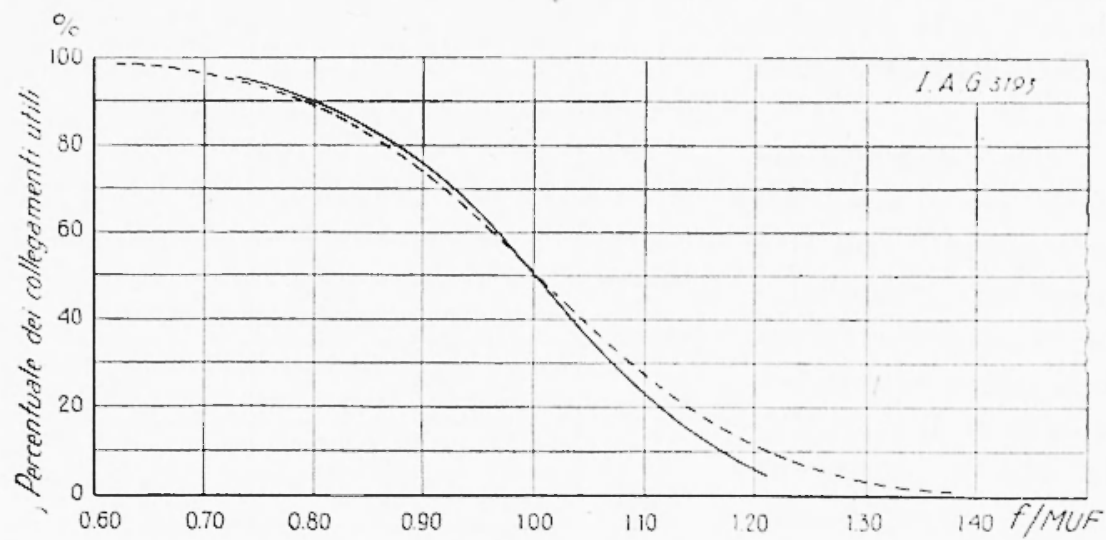

Fig. j. Distribuzione nedia rieasala dalle esperienze. Lat rurval a tratlo pieno è la stessil della fig. f. Quella tratteggiatla corrisponde ad una di-tribuziom. gatussianil di searto medio uguale a quella ottemuta dalle esperienze.

senta una distrihuzione gaussiana di ampiezza uğuale a quella media (cfr. tabella IV).

Se si fa rariare la frequenza al di sotto della MLF si trova greneralmente una frequenza oltima. La frequenza oltima non supera nai $0,9 \mathrm{C}$, e spesso è inferiore a $0.8 \mathrm{C}$. Secondo la distribuzione di fig. 5 che non tiene conto dell'assorlimento, in media il coefficiente da applicare alla MUF per otlenere ma frequenza che dia il $95 \%$, di colleganenti utili è 0,73 , invece del 0.85 usato per calcolare la FOT.

5. Attendibilità dei risultati. - 5.1. - Complessivamente sono state considerate oltre 60.000 ricezioni effeltuate generalnente ad intervalli di 1 ora, da 12 stazioni radio e da un numero alneno triplo di narconisti. I dati otlenuti rappresentano perio ahbatsanza fodelmente il comportamento medio di una rele R.T. delle caralteristiche di ruella studiata, cioe: 
-- fumzionamento in radiolelegrafia a trasmissione manuale e ricozione in culfia;

-- impianto di iino quasi-campale;

- dislanze comprese tra 200 e $600 \mathrm{~km}$, nella zona lemperata.

5.2. - Fi però ulile elencare esplicitamente le ipolesi assunte a hase del lavoro:

5.2.1. - Come legge di variazione della MLF nella giornata e nell'amo si a as-anta quella data dal CRPL. Alla juce di sucessive ricerche lale legge risulta valida solo in prima approssimazione.

5.2.2. - Alla base del metodo sta l'ipotesi che le fluttuazioni della MLif la un giorno all'altro nel mese sieno proporzionali alla IILF mediana di ciaseuna ora. Questo in realta non aviene. I dati medi otlenuti sono peris ancora validi, con qualehe linitazione.

5.2.3. ... Si i ammesso che la ionizzazione sia uguale in lutli $i$ punti di riflesione comsicierati. In realta le differenze delle frequenze rritiche superano solo raramente il $t \%$, ma in pochi minuli dopo l'allat, nel mese di dicembre, si ha una differenza massima magrgiore del $20 \%$.

6. Comrlasioni, - E possibile trare ora le seguenti conclusioni, valide per eomelizioni analoghe a quelle di sperimentazione, e di cui aleune sono state anticipate nella parte 1.

6.1. - La probabiliti di un ascolto riuscito $10 \mathrm{~S} />01$ senzal he il collegamento sia utilizzabile ogSt > 2) $\mathrm{e}$ molo alta, specialmente alle frequenze più alte. Ne risulta necessaria la norma prulenziale di coltatulare qualunque collegamento con la clfeltiva trasmi-sione di messageri.

6.2. - Quando la frequenza ammenta variando intorno alla IILF la perentuale dei collegancnti utili diminuisece con una distribuzione ehe differise poco da quella alrateria normale, ed è data dal diagre. 5.

La distribuzione delle perentuali dei sinğoli collegamenti è molto slarsa: percio non si fuo fare alcun aflidamento su esperienze sin-

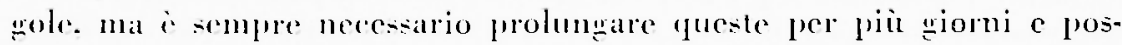
silibmente $\$ 11$ più stazioni. 
6.3. - Generalmente esiste una frequenza obluma, che $i$ sempre minore della MUF (per le condizioni di sperimentarione essa d ninore di $0,85 \quad M L F_{\text {co. }}$ In corrisnondenza della lreguenza ottima una variazione di potenza da 40 a 1000 wall riduee nel rapporto 1 a l la probahilita di collegamenti non utilizzaliali (da 9,9 a $2,6 \%$ ).

6.4. - A prescinlere da questa probabilita di collegamenti non mlilizzahili dovula a defecienza di potenza, la frequenza mediana va ridotta secondo un coefficiente 0,73 per avere la frequenza che da una probabilita del 95\%. Adothando il coefficiente di riduzione 0,85, che ¿ quello raccomantato dal CRPL per ottenere la FO'T, la probahiliti i dell'85\% soltanto.

Resta infme confermala la necessitat di trare sli elementi per il progetto dei collegamenti radio da misure else possano distinguere $i$ singroli fattori, in quanto i dati ottentuti dallesercizio richiedono wranle dispentio di lavoro e molto tempo per la loro interpretazione, e anche l'esame di un gran numero di dati lascia forti incertezze. Cii puì essere griustificato quando non si dispongra di ionosonde o quando, come nel presente studio, si voglia procedere a confronti. i anche necessario il confronto tra misure e dati di esercizio nello studio di problemi nuovi, come quello della predeterminazione della sienrezza o grato di affrdamento ("relisthility") dei collegamenti radio, recentemente impostato dalla Commissione $6^{\mathrm{a}}$ del C.C.I.R.

$$
\begin{aligned}
\text { Roma - Istituto Nazionale di Geofisica. } & \\
& \text { Ispettorato dell'Arma del Genio -- Lumlio 19.50. }
\end{aligned}
$$

\section{BIBI.IOGR:IFIA}

J. В. Scановоскн: Numerical Mathematical Analysis. Oxford 1930.

G. Castelsuovo: Calcolo delle probabilitio. Vol. I. Zanichelli, Bologna 1917. Nittional Bureau of Standards. Circ. 165. Washington $191 \%$.

R. A. Fismer: Metodi Statistici. UTFT. Torino $19 \pm 8$.

National Bureau of Standards. Circ. 162: Ionospherir Radio Propuration. Washington 1918.

National Bureau of Standards. Basic Radio Propagation Predictions i D seriesi. Mensile.

A. Bolde, S. Sultexi e C. A. Tubno: Registrazioni ionosferiche. Annali di Geofisical, Yol. II, n. 3, pagg. 377.387 (luglio 1919). 